\title{
Flower Classification Using Neural Network Based Image Processing
}

\author{
Dr.S.M.Mukane ${ }^{1}$, Ms.J.A.Kendule ${ }^{2}$ \\ ${ }^{1}$ (Electronics \& Telecommunication, SVERI's College of Engineering, Pandharpur, India) \\ ${ }^{2}$ (Electronics \& Telecommunication, SVERI's College of Engineering, Pandharpur, India)
}

\begin{abstract}
In this paper, it is proposed to have a method for classification of flowers using Artificial Neural Network (ANN) classifier. The proposed method is based on textural features such as Gray level co-occurrence matrix (GLCM) and discrete wavelet transform (DWT). A flower image is segmented using a threshold based method. The data set has different flower images with similar appearance. The database of flower images is a mixture of images taken from World Wide Web and the images taken by us. The ANN has been trained by 50 samples to classify 5 classes of flowers and achieved classification accuracy more than 85\% using GLCM features only.
\end{abstract}

Keywords: Artificial Neural Network, DWT, GLCM, Segmentation.

\section{Introduction}

Developing a system for classification of flowers is a difficult task because of considerable similarities among different classes. In a real environment, images of flowers are often taken in natural outdoor scenes where the lighting condition varies with the weather and time. Also, there is lot more variation in viewpoint of flower images. All these problems lead to a confusion across classes and make the task of flower classification more challenging. In addition, the background also makes the problem difficult as a flower has to be segmented automatically.

Applications of classification of flowers can be found useful in floriculture, flower searching for patent analysis, etc. The floriculture industry comprises flower trade, nursery and potted plants, seed and bulb production, micro propagation, and extraction of essential oil from flowers. In such cases, automation of flower classification is essential. Since these activities are done manually and are very labor intensive, automation of the classification of flower images is a necessary task.

\section{Previous Work}

S. Manjunath et.al [2] has investigated the effect of texture features for the classification of flower images using probabilistic neural network as a classifier. S.M.Mukane et.al [3] has presented DWT and GLCM based feature selection for scale invariance texture image retrieval using fuzzy logic classifier. S.M.Mukane et.al [5] has presented wavelet and cooccurrence Matrix based features for rotation invariant texture image retrieval using fuzzy logic classifier. Saitoh et al. [6] designed a flower classification system which extracts features from both flowers and leaves, and used a piecewise linear discrimant analysis for recognition on a dataset of 34 species each containing 20 sets of wild flowers. M. Das et al. [7] proposed an indexing method to index flower patent images using domain knowledge. Each flower image is discredited in HSV color space, and each point on the discredited HSV space is mapped to a color name in the ISCC-NBS and X Window systems in order to index the flowers. Nilsback and Zisserman [8] designed a flower classification system by extracting visual vocabularies which represent the color, shape, and texture features of flower images. Nilsback and Zisserman [9] noted that color and shape are the major features in flower classification. In this work, we investigate the suitability of texture features in designing a system for flower classification. Nilsback and Zisserman in their work [10] considered a dataset of 103 classes, each containing 40 to 250 samples. The lowlevel features such as color, histogram of gradient orientations, and SIFT features are used. They have achieved an accuracy of $72.8 \%$ with an SVM classifier using multiple kernels. Nilsback and Zisserman [11] proposed a two-step model to segment the flowers in color images, one to separate the foreground from background and the other to extract the petal structure of the flower. This segmentation algorithm is tolerant to changes in viewpoint and petal deformation, and the method is applicable in general for any flower class. Yoshioka et al. [12] performed a quantitative evaluation of petal colors using principal component analysis. They considered the first five principal components (PCs) of a maximum square on the petals. D.S. Guru et al [13] have proposed an algorithmic model for automatic classification of flowers using KNN classifier. In their work, Saitoh et al. [14] describe an automatic method for recognizing a blooming flower based on a photograph taken with a digital camera in a natural scene. It is based on "Intelligent Scissors" [15], which find the path between two points that minimizes a cost function dependent on image gradients. 


\section{Our Approach}

The proposed method has training and classification phases. In the training phase, from a given set of training images (segmented) the texture features (DWT and GLCM) is extracted. This extracted texture features are used to train the system using a Multilayer Perceptron Neural Network.

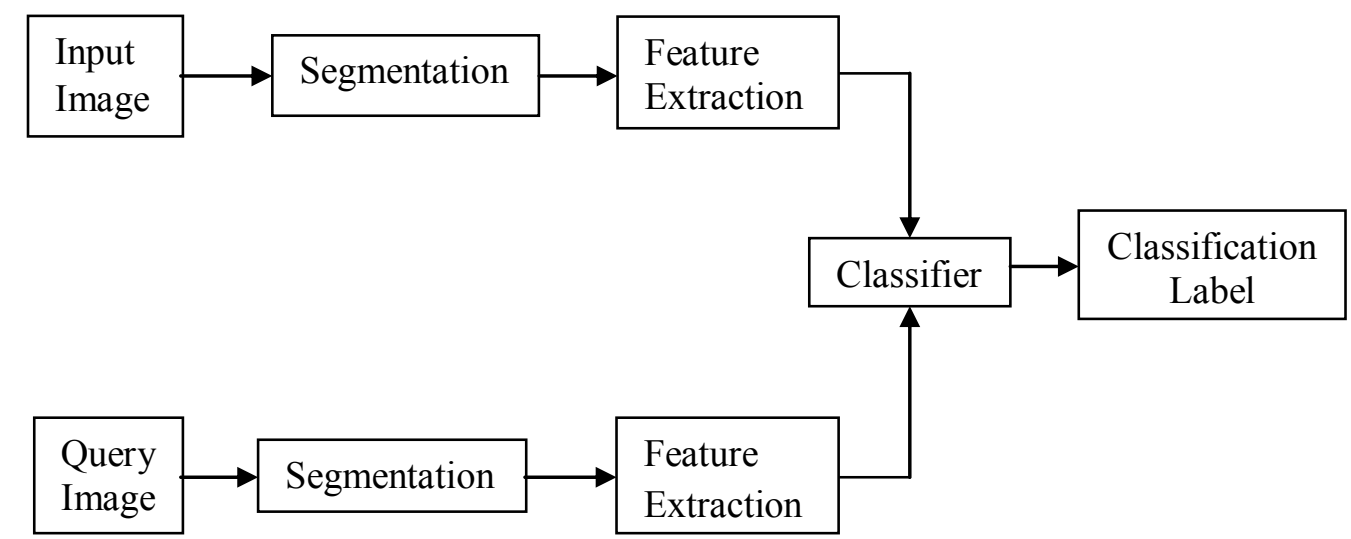

Fig 1: Block diagram of the proposed work.

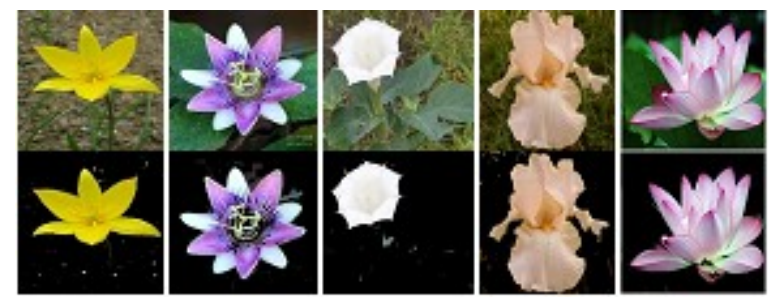

Fig. 2 Segmentation result (a) Input images and (b) segmented images.

In the classification phase first all the flowers is segmented from test image set and then texture features are extracted. These features are queried to the Multilayer Perceptron neural network to know the flower class. The block diagram of the proposed method is given in Fig. 1.

The first step in flower classification is to segment the flower image. In this step from flower image remove the unwanted background region. Flowers in images are generally surrounded by greenery in the background [16]. In order to avoid matching the green background region, rather than the desired foreground region, the image has to be segmented. To segment the flower image, we use a semi-automated threshold-based segmentation algorithm. Fig. 2 shows the results of flower segmentation using the threshold-based method on a few sets of images with a cluttered background.

\section{Feature Extraction}

Certain products are easily identified by simply color, for example, jowar and ground nut, pomegranate and mango etc and color becomes the discriminating feature. But some have overlapping colors, for example, wheat and ground nut, mango and orange etc. When we consider the bulk samples of such products, the surface patterns vary from sample to sample. In such cases, the texture becomes ideal for recognition. Hence, we have obtained textural features of the image samples to recognize and classify the flower. The textural features namely energy and standard deviation are extracted using DWT at forth level decomposition.

\subsection{DWT}

Traditionally, Fourier transforms have been utilized for signal analysis \& reconstruction. However, Fourier transform does not include any local information about the original signal. Therefore, Short Time Fourier Transform (STFT or Gabor transform) has been introduced, which uniformly samples the timefrequency plane. Unlike the STFT which has a constant resolution at d times and frequencies, the wavelet transform has a good time and poor frequency resolution at high frequencies, and good frequency and poor time resolution at low frequencies.

In JPEG2000, Discrete Wavelet Transform is used as a core technology to compress still images. The DWT has been introduced as a highly efficient and flexible method for sub band decomposition of signals [13]. The two dimensional DWT (2D-DWT) is nowadays established as a key operation in image processing. It is multi-resolution analysis and it decomposes images into wavelet coefficients and scaling function. In Discrete 
Wavelet Transform, signal energy concentrates to specific wavelet coefficients. This characteristic is useful for compressing images.

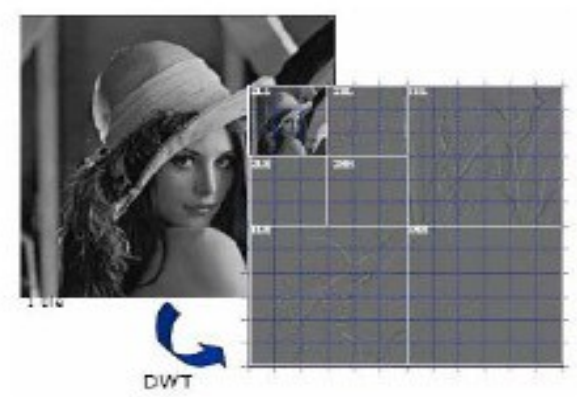

Fig. 3 Two- level Decomposition of Image

Fig. 3 shows 2- level DWT decomposition. Highest level of decomposition depends upon the wavelet filter used, need of the application and features required for the classification. Using $4^{\text {th }}$ level of DWT decomposition, coefficients of approximate \& detail Sub-bands are extracted. Based on the available wavelet coefficients, Energy (1) and standard deviation (2) of all the sub-bands up to fourth level of decomposition are calculated.

$$
\begin{aligned}
& E_{k}=\frac{1}{N} \sum_{i=1}^{N} \sum_{j=1}^{N}\left|x_{k}(i, j)\right| \\
& \sigma_{k}=\left[\frac{1}{N^{2}} \sum_{i=1}^{N} \sum_{j=1}^{N}\left(x_{k}(i, j)-\mu_{k}(i, j)\right)\right]^{\frac{1}{2}}
\end{aligned}
$$

Where $E_{k}$ is the energy \& $\sigma_{k}$ is the standard deviation for the kth sub-band of dimension NxN and coefficients are $X_{k}(i, j) \&$ mean value is $\mu_{k}(i, j)$. For each samples of different class image, above features are computed and stored in the data base feature vector as Wavelet Statistical Features (WSF). This feature is used at the time of classification stage.

\subsection{GLCM}

Texture feature calculations use the contents of the GLCM to give a measure of the variation in intensity at a pixel of interest. First proposed by Haralick et al. [13] in 1973, they characterize the texture using a variety of quantities derived from second-order image statistics. Co-occurrence texture features are extracted from an image in two steps. First, the pair wise spatial co-occurrences of pixels separated by a particular angle and distance are tabulated using a gray level co-occurrence matrix (GLCM). Second, the GLCM is used to compute a set of scalar quantities that characterize different aspects of the underlying texture. The GLCM is a tabulation of how often different combinations of gray levels co-occur in an image or image section [13].

The GLCM is an $\mathrm{N} \times \mathrm{N}$ square matrix, where $\mathrm{N}$ is the number of different gray levels in an image. An element $p(i, j, d, \theta)$ of a GLCM of an image represents the relative frequency, where $i$ is the gray level of pixel $\mathrm{p}$ at location $(\mathrm{x}, \mathrm{y})$ and $\mathrm{j}$ is the gray level of a pixel located at a distance $\mathrm{d}$ from $\mathrm{p}$ in the orientation $\theta$. While GLCMs provide a quantitative description of a spatial pattern, they are too unwieldy for practical image analysis. Haralick et al. [13] thus proposed a set of scalar quantities for summarizing the information contained in a GLCM. They originally proposed a total of 14 quantities, or features; however, typically only subsets of these are used [14].

The eight GLCM-derived features inverse difference moment, contrast, local homogeneity, energy, norm entropy, cluster shade, cluster prominence, maximum probability are extracted and used in this work.

$$
\begin{aligned}
& \text { Inverse difference moment }=\sum_{\substack{i=1 \\
i \neq j}}^{N} \sum_{j=1}^{N} \frac{\operatorname{Co}(i, j)}{|i-j|^{2}} \\
& \text { contrast }=\sum_{i=1}^{N} \sum_{j=1}^{N}|i-j|^{2} \operatorname{Co}(i, j) \\
& \text { energy }=\sum_{i=1}^{N} \sum_{j=1}^{N} \operatorname{Co}^{2}(i, j)
\end{aligned}
$$




$$
\begin{aligned}
& \text { norm entropy }=\frac{\sum_{i, j=1}|\operatorname{Co}(i, j)|^{1.5}}{N} \\
& \text { local homogeneity }=\sum_{i, j=1}^{N} \frac{1}{1+(i-j)^{2}} \operatorname{Co}(i, j) \\
& \text { cluster shade }=\sum_{i, j}^{N}\left((i-P x+j-P y)^{3}\right)(\operatorname{Co}(i, j)) \\
& \text { cluster prominance }=\sum_{i, j}^{N}\left((i-P x+j-P y)^{4}\right)(\operatorname{Co}(i, j)) \\
& \text { maximum probility }=\operatorname{Max}[\operatorname{Co}(i, j)] \\
& P_{x}=\sum_{i, j=1}^{N} i \operatorname{Co}(i, j) \text { and } P_{y}=\sum_{i, j=1}^{N} j \operatorname{Co}(i, j)
\end{aligned}
$$

For each samples of different class image, above features are computed and stored in the data base feature vector as GLCM features. Therefore these 8 features are used at the time of classification stage.

\section{Flower Classification (Ann)}

Artificial neural network (ANN) as a classifier has been used. An Artificial Neural Network (ANN) is an information-processing paradigm that is inspired by the way biological nervous systems, such as the brain, process information.

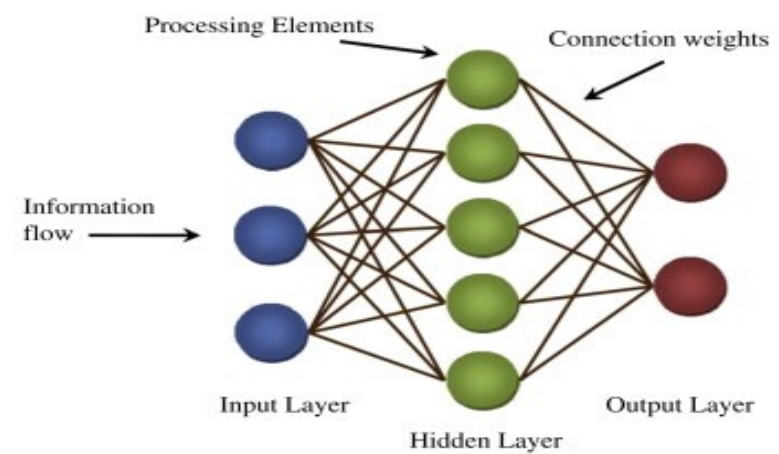

Fig.4 The general architecture for MLP networks

The MLP and many other neural networks learn using an algorithm called backpropagation. With backpropagation, the input data is repeatedly presented to the neural network. With each presentation the output of the neural network is compared to the desired output and an error is computed. This error is then fed back (back propagated) to the neural network and used to adjust the weights such that the error decreases with each iteration and the neural model gets closer and closer to producing the desired output. The general architecture for MLP networks is shown in Fig.4.

Performance of the above feature sets is tested with the help of a MLP classifier in terms of Success rate. Let PT be the no. of samples to be tested and out of that the system correctly classifies is PC then percentage is given as:

$$
\mathrm{Pr}=\mathrm{PC} / \mathrm{PT} * 100
$$

\section{Experimental Results and Discussions}

In this work our own database inspite of existence of other databases has been created. Flower images from World Wide Web in addition to images from own database that can be found in and around the area. The database consists of 5 classes of flowers with 10 images of each. The images are rescaled to the size 256x256 using bicubic interpolation.

In this experiment pyramid structured type of DWT is used with dB8 as a wavelet filter. Feature database is created using wavelet decomposed subbands up to forth level of decomposition. Total number of subbands up to forth level will be 16. Energy (1) and standard deviations (2) of each sub-band coefficients are calculated for each level of the samples.

From the co-occurrence matrix the co-occurrence parameters namely contrast, inverse difference moment, energy, norm entropy, local homogeneity, cluster shade, cluster prominence, \& maximum probability are obtained. These features are stored in database vector. 


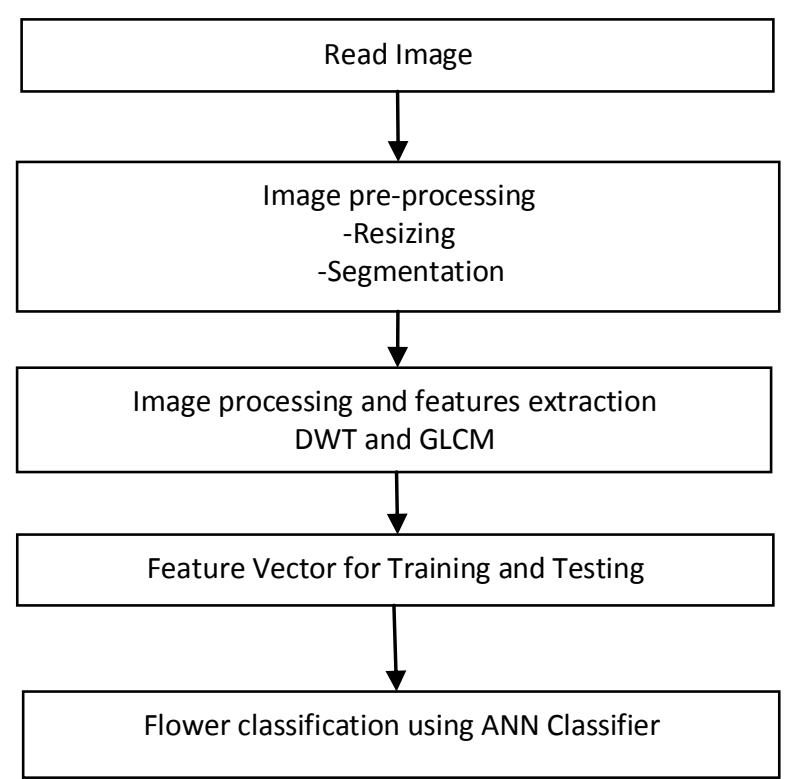

Fig. 5 Flowchart for Flower Classification

Experimentation has been conducted on databases of 50 images and 5 classes. The classification accuracy under ANN classifier has been investigated. As compared with other work we have used only gray level features and not used any color feature.

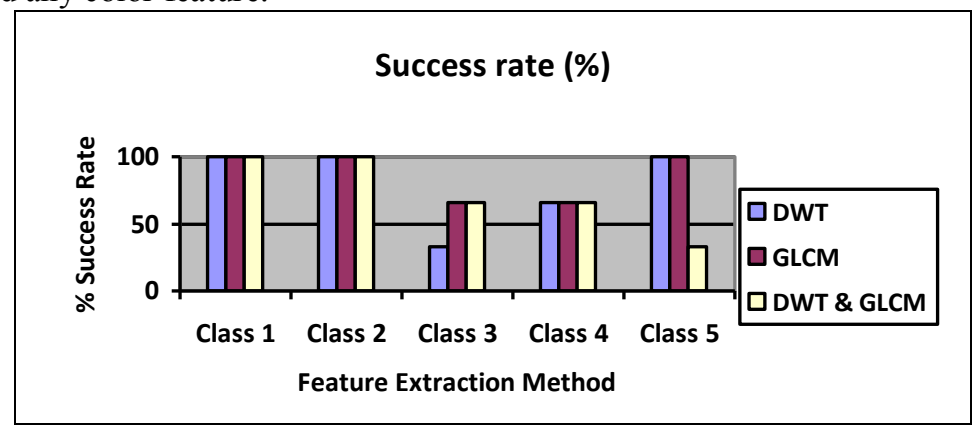

Fig.6 Success Rate (\%) with each class

Figure 6 shows success rate for every class with different features (DWT and GLCM) with sample size of $256^{*} 256$. As compared with all classes GLCM features shows highest result while combination of DWT and GLCM shows less success rate. Hence it is found that flower images can be classified easily with the GLCM features only.

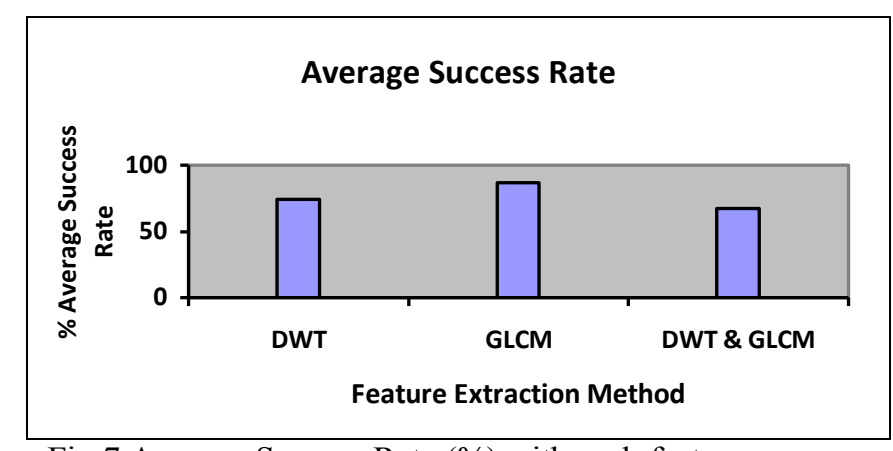

Fig.7 Average Success Rate (\%) with each feature

\section{Conclusion}

Image processing technique plays an important role in the classification. The use of a neural network classifier for flower classification using DWT and GLCM has been demonstrated. Only gray level features have been used. The neural network is trained using the backpropagation algorithm. Own database of flowers of 5 
classes, each containing 10 flower images has been created. It has been found that MLP offers accuracy $87 \%$ with GLCM features.

\section{References}

[1]. D.S. Guru, Y.H. Sharath Kumar, S. Manjunath, "Textural features in flower classification", Mathematical and Computer Modeling Journal, 2010

[2]. Dayanand G. Savakar and Basavaraj S. Anami, "Recognition and Classification of Food Grains, Fruits and Flowers Using Machine Vision", International Journal of Food Engineering, Volume 5, Issue 4, Article 14, Pages: 1-25,

[3]. Shailendrakumar M.Mukane, Sachin R. Gengaje, Dattatraya S. Bormane," On Scale Invariance Texture Image Retrieval using Fuzzy Logic and Wavelet Co- occurrence based Features", International Journal of Computer Applications (0975 - 8887) Volume 18No.3, March 2011

[4]. Shailendrakumar M.Mukane, Sachin R. Gengaje, Dattatraya S. Bormane "Wavelet and Co-occurrence Matrix based Rotation Invariant Features for Texture Image Retrieval using Fuzzy Logic”, International Journal of Computer Applications (0975 - 8887) Volume 24- No.7, June 2011

[5]. B. S. Anami, Dayanand G. Savakar, "Effect of Foreign Bodies on Identification and Classification of Bulk Food Grains Image Samples", Journal of Applied Computer Science and Mathematics, Volume 3(6), Pages: 77- 83.

[6]. T. Saitoh, T. Kaneko, in: 15th International Conference on Pattern Recognition, ICPR'00, and pp. 507-510.

[7]. M. Das, R. Manmatha, E.M. Riseman, IEEE Intelligent Systems 14 (1999) 24-33.

[8]. Nilsback Maria-Elena, A. Zisserman, in: CVPR'06: Proceedings of the 2006 IEEE Computer Society Conference on Computer Vision and Pattern Recognition, pp. 1447-1454.

[9]. Y.Y. Boykov, M.P. Jolly, in: Eighth IEEE International Conference on Computer Vision, 2001, ICCV 2001, Proceedings, vol. 1, pp. $105-112$.

[10]. Nilsback M. Elena, Z. Andrew, in: ICVGIP'08: Proceedings of the 2008 Sixth Indian Conference on Computer Vision, Graphics and Image Processing, pp. 722-729.

[11]. Nilsback Maria-Elena, Z. Andrew, Image and Vision Computing 28 (2010) 1049-1062.

[12]. Y. Yoshioka, H. Iwata, R. Ohsawa, S. Ninomiya, Euphytica 139 (2004) 179-186.

[13]. M. Varma, D. Ray, in: IEEE 11 th International Conference on Computer Vision, pp. 1-8.

[14]. T. Saitoh, K. Aoki, T. Kaneko, in: 17th International Conference on Pattern recognition, ICPR'04, pp. 27-30.

[15]. E.N. Mortensen, W.A. Barrett, in: SIGGRAPH'95: Proceedings of the 22nd Annual Conference on Computer Graphics and Interactive Techniques, pp. 191-198.

[16]. R.C. Gonzalez, R.E. Woods, S.L. Eddins, Digital Image Processing Using MATLAB,Prentice-Hall, Inc., 2003. 